\title{
Clearing Deleterious Proteins for Healthier Aging
}

\author{
Ian Macreadie, Sudip Dhakal \\ School of Science, RMIT University, Melbourne, Australia \\ Email: ian.macreadie@rmit.edu.au
}

How to cite this paper: Macreadie, I. and Dhakal, S. (2019) Clearing Deleterious Proteins for Healthier Aging. Open Journal of Social Sciences, 7, 128-132. https://doi.org/10.4236/jss.2019.75010

Received: April 10, 2019

Accepted: May 14, 2019

Published: May 17, 2019

\begin{abstract}
The build-up of deleterious proteins is one of the biggest problems in aging. The brain, the organ most sensitive to this phenomenon, seeks to maintain the correct balance by the process known as proteostasis but this process declines with aging. Reduced proteostasis causes major age-related diseases such as Alzheimer's Disease, Parkinson's Disease, and prion diseases, where aberrant proteins are known to associate with such diseases. Being able to restore proteostasis is likely to slow age-related decline in brain function, but the challenges are to find chemo preventatives that can enhance proteostasis to youthful levels, and to know how to administer these chemopreventative agents. A combination of epidemiology and studies in a convenient model system are providing approaches to find answers to these important questions.
\end{abstract}

\section{Keywords}

Alzheimer's Disease, Autophagy, Protein Folding, Protein Turnover, Proteostasis, Saccharomyces cerevisiae, Simvastatin

\section{Introduction}

The correct assembly of cells and their maintenance is intrinsic to survival and healthy aging. This process, known as homeostasis, is fundamental and cells have a large variety of approaches, some totally dedicated to this work, to maintain the necessary equilibrium. Without the balance a cell might ultimately die, or in the shorter term become dysfunctional leading to the breakdown of a network where it normally functions. There is indeed a parallel between this and other networks like computing systems or transport networks, that are very familiar to us. The loss of any one small component may lead to a breakdown of the entire system. 
A sub part of homeostasis is proteostasis, the turnover of proteins. Proteins are the diverse machinery of cells that include enzymes, antibodies, structural proteins and components of the complex systems that enable cells to replicate and respond to cellular needs. The cell has the potential to produce tens of thousands of proteins to meet its needs and these proteins can be present within the cell at concentrations of $500 \mathrm{mg} / \mathrm{ml}$. This is an incredibly high concentration of matter, and one which cannot be achieved outside the cell by any human technology. The high density inside the cell requires precise folding of proteins so that they can be close packed and yet retain their function.

Things are so critical inside the cell that systems exist to degrade proteins too. This degradation is not totally random: different proteins have different half-lives. That is, they are degraded at different rates. Physiology too can play a role. If cells are rapidly dividing the focus may be on protein synthesis and protein levels might be maintained by cell division. For example, if proteins are doubled at the same rate as cell division, there is equilibrium. However, many cells in our body have very low rates of cell division and many, especially most brain cells, have stopped dividing. Nevertheless, if they are to continue to perform essential brain functions they must continue to live for decades. Their challenge is huge: they need to maintain proteostasis, or risk death or dysfunction. There is a decline in proteostasis with aging, and in the brain this is most evident, and accounts for major age-related neurodegenerative diseases like Alzheimer's disease and Parkinson's disease.

A number of processes contribute to protein turnover and some of these are described below.

\section{Proteostasis}

\subsection{Autophagy}

The 2016 Nobel Prize was awarded to Yoshinori Ohsumi for his work on the aspect of proteostasis known as autophagy, highlighting how important proteostasis is. Autophagy [Greek: self-eat] refers to the process where the cell degrades unwanted proteins. It may do this through the use of a range of proteases.

Autophagy is a crucial process in eukaryotic cells by which the unwanted larger structures, misfolded proteins and their aggregates are cleared from the cells by the formation of a large double membrane autophagosome that delivers this cargo into lysosomes forming autolysosomes. The highly conserved process declines as ageing progresses. Ageing comes with changes in the post translational modifications, chromatin remodelling, ROS accumulation creating highly oxidative environment, structural and functional change of the proteins, altered levels of metabolites, altered levels of biometals, all of which have been found impacting the homeostasis of the cells either directly or indirectly.

\section{Mitophagy}

Mitophagy refers to the turnover of mitochondrial proteins. The mitochondrion generates the energy required by cells and during its respiration it generates ROS 
which can be deleterious. Hence it is important to have mechanisms to protect against ROS and to clear damaged proteins in the mitochondrion so that it can continue its respiratory function. Respiratory functions decline considerably with ageing.

\subsection{Unfolded Protein Response}

Intracellular aberrant/misfolded protein aggregation leading to proteostatic stress induces a stress response in the endoplasmic reticulum to clear deleteriouss proteins. Thus is also referred to as the unfolded protein response. Cells under stress try to retain their protein balance by inhibiting the protein synthesis mechanism, inducing the protein folding by upregulating chaperones and foldases, and finally by directly clearing them from the endoplasmic reticulum. It has also been shown that with ageing the efficiency of unfolded protein response decreases.

Under certain conditions, such as high temperature, proteins may become denatured or misfolded, leading to a response often called the "heat shock response". The heat shock response has been well studied in the unicellular eukaryotic microbe yeast, where around 50 proteins are produced in response to elevated temperature to deal with misfolded proteins. Other conditions that may lead to this response are exposure to reactive oxygen species (ROS), and some proteins through mutation or their intrinsic structure may be prone to aggregation. For example, the poly glutamine repeats in the Huntingtin protein inevitably lead to Huntington's disease.

\subsection{Ubiquitin Proteasome System}

The ubiquitin proteasome system is another highly conserved system that governs the protein balance within cells. Misfolded and damaged protein are recognized and degraded by tagging them with the poly-ubiquitin tags. The ubiquitin tags allow the protein clearance machinery to recognize and clear the tagged molecules. This mechanism is also found to be impaired with ageing and is also found to cause age-associated pathology and shortened lifespan in flies and mice [1] [2].

\section{Why Do We Have Reduced Proteostasis with Age?}

This question is like the chicken and egg question. Which one comes first? While there is the high degree of association between the two, there appears to be no answer so far. However, as we learn more from interventions in proteostasis we may start to get answers. Possibly a lifetime of chemical insults may add up to causing a non-reversible decline. We are examining the effects biogenic amines, natural biochemicals that we produce, to determine how they may affect cellular functions.

\section{Yeast as a Model for Studying Aging and Interventions}

Budding yeast cells can serve as a good model to study aging. In a population of 
budding yeast (shown in Figure 1), half of the population is newly budded and young, while the cells that have given rise to this population are first time mothers (25\% of the population), grandmothers delivering their second budded cell (12.5\%), and even older cells delivering their third (6\% of the population), fourth ( $3 \%$ of the population) or greater buds ( $1 \%$ of the population). The bud scars serve as a good indicator of the age of cells and they can be specifically stained with calcofluor enabling the different cell types to be distinguished. Cell size is another indicator of yeast age: cells get progressively bigger with age.

We have developed yeast as a model to examine the decline in proteostasis with age. This has been done by examining the proteostasis of the Alzheimer's amyloid beta $(\mathrm{A} \beta)$ protein. This protein accumulates with age and is highly associated with Alzheimer's Disease. In human populations that don't develop Alzheimer's Disease A $\beta$ is negligible in amount. However, in early onset Alzheimer's Disease people produce $\mathrm{A} \beta$ in excessive amounts. This protein has occupied more attention from researchers than other proteins in Alzheimer's Disease research, however, treatments that remove $\mathrm{A} \beta$ in people with Alzheimer's Disease do not cure the disease, leading many to give up on strategies to deal with $\mathrm{A} \beta$.

To readily observe $\mathrm{A} \beta$ in real time we made genetically engineered yeast that constitutively produce Alzheimer's A $\beta$ fused to a green fluorescent protein (GFP, from a jellyfish) [3]. Although all cells produce the protein, the A $\beta$ fused to GFP is recognised as an undesirable protein and is degraded by proteostasis mechanisms in all cells except the oldest ones in the population. So around 10\% - 25\% of the cells in the population exhibits green fluorescence. We have studied such populations to screen for compounds that reduce the levels of green fluorescence in the old cells. This may offer new leads into drug classes that improve proteostasis.

\section{Simvastatin}

One pharmaceutical compound that has shown benefits for the prevention of Alzheimer's Disease is the cholesterol-lowering drug simvastatin. In their 2007
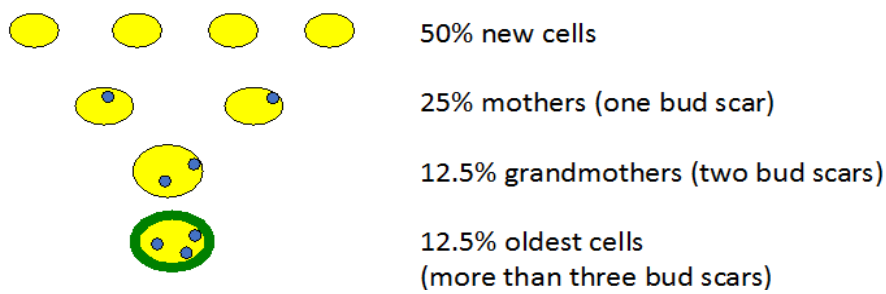

Figure 1. Schematic representation of a budding yeast population. All cells can divide and bud off a new cell which is distinguished as being slightly smaller and having no bud scars. Each budding event leaves a scar on mother cells so that their age can be readily discerned by visual microscopic examination or other means, including staining of bud scars followed by flow cytometry. The oldest cells in the population above have 3 or more scars and exhibit signs of aging. 
epidemiology study involving examination of health outcomes in millions of statin users in the Veteran's Administration program, Wolozin and colleagues found that simvastatinled to a 50\% reduction in Alzheimer's Disease and Parkinson's Disease [4]. In contrast, other statins like atorvastatin and lovastatin, did not have a significant effect on Alzheimer's Disease and Parkinson's Disease.

Just how simvastatin exerts this protective effect has not been clear. For example, is it through cholesterol lowering? Our studies in yeast have shown that simvastatin aids clearance of amyloid beta in a manner than appears independent of cholesterol lowering. Although yeast does not produce cholesterol it has an equivalent pathway that includes the HMG-CoA reductase (the target of statins) to produce ergosterol which is functionally equivalent to cholesterol. Utilising fluconazole to lower ergosterol did not lead to clearance of amyloid beta. It would appear that simvastatin may have an "off target' effect that is to improve proteostasis of amyloid beta, and this may lead to the $50 \%$ reduction in Alzheimer's Disease seen in Wolozin's study.

\section{Conclusions}

Declining proteostasis with ageing is a considerable concern, particularly when it affects our most sensitive organ, the brain.

In addition to finding efficient new drugs, even better than simvastatin, that enable clearance of deleterious proteins in the elderly, consideration needs to be given to nutraceuticals and foods that are acceptable throughout life as part of our diets. On the other side, consideration also needs to be given to discovering factors that may enhance the decline in proteostasis with aging, so that these can be avoided.

\section{Conflicts of Interest}

The authors declare no conflicts of interest regarding the publication of this paper.

\section{References}

[1] He, L.Q., Lu, J.H. and Yue, Z.Y. (2013) Autophagy in Ageing and Ageing-Associated Diseases. Acta PharmacologicaSinica, 34, 605-611. https://doi.org/10.1038/aps.2012.188

[2] Sands, W.A., Page, M.M. and Selman, C. (2017) Proteostasis and Ageing: Insights from Long-Lived Mutant Mice. Journal of Physiology, 595, 6383-6390. https://doi.org/10.1113/JP274334

[3] Caine, J., Sankovich, S., Antony, H., Waddington, L., Macreadie, P., Varghese, J. and Macreadie, I. (2007) Alzheimer's A $\beta$ Fused to Green Fluorescent Protein Induces Growth Stress and a Heat Shock Response. FEMS Yeast Research, 7, 1230-1236. https://doi.org/10.1111/j.1567-1364.2007.00285.x

[4] Wolozin, B., Wang, S.W., Li, N.C., Lee, A., Lee, T.A. and Kazis, L.E. (2007) Simvastatin Is Associated with a Reduced Incidence of Dementia and Parkinson's Disease. BMC Medicine, 5, 20. https://doi.org/10.1186/1741-7015-5-20 